Condensed Matter Physics, 1999, Vol. 2, No. 1(17), p. 143-148

\title{
Conductivity of layered structures with a strongly degenerate electron gas
}

\author{
P.P.Petrov \\ State University "Lvivska Politechnika", \\ 12 S.Bandera Str., 290646 Lviv, Ukraine
}

Received August 31, 1998

\begin{abstract}
The conductivity of periodical multilayer structures with a degenerate electron gas is calculated. It is shown that for smooth tunnelly transparent barriers the resistivity of structure coincides with the corresponding value of the bulk material. In the presence of a random potential at the interface between the layers, the conductivity of the system decreases with the increase of a ratio of the mean free path to the period of the structure.
\end{abstract}

Key words: multilayer structure, conductivity.

PACS: $73.20 . H, 82.65 . Y$

The study of the effect of the boundaries on the electrical conductivity of thin films is a well-known problem of solid state physics. An interest in it is aroused due to the latest intense investigations of multilayer periodical structures with the conductivity of a metallic type. When considering the conductivity of spatially restricted systems on the basis of the Boltzman equation, the problem of correct boundary conditions for distribution functions is of essential importance.

We consider a structure consisting of many layers of the same material with a degenerate electron gas, divided either by thin tunnelly transparent barriers or by planes with randomly distributed scattering centres on them. This role can be played by any defects of the interface. A similar situation is observed, for example, in metallic superlattices or semiconductor structures with delta-doped layers. The width of the conducting layers $z_{0}$ is assumed to be large enough comparing to the electron wavelength $\lambda$, so that the effect of size quantization could be neglected. To calculate the conductivity of the structure we use a method based on the solution of a kinetic equation in the layer with periodical boundary conditions. At the interface the boundary conditions for the distribution functions are determined by joining the electron wave functions.

Let the electric field $E=(E, 0,0)$ be directed along the conducting layers plane which is perpendicular to the z-axis of the structure. The kinetic equation for distribution functions inside each layer under a weak electric field has the form 


$$
e E v_{x} \frac{\partial f_{0}}{\partial \epsilon}+v_{z} \frac{\partial f}{\partial z}+\frac{f-f_{0}}{\tau}=0
$$

and takes into account the scattering from impurities in the bulk of the layer within the framework of the relaxation time $\tau$ approximation. Here $f_{0}$ is an equilibrium distribution function.

Let us consider first an electric current transport in the layer $0<z<z_{0}$. The solution of equation (1) can be written separately for carriers moving along the axis of the structure and in the opposite direction:

$$
\begin{gathered}
f^{+}=f_{0}+C_{1} \mathrm{e}^{-z / l}-e E v_{x} \tau\left(1-\mathrm{e}^{-z / l}\right) \frac{\partial f_{0}}{\partial \varepsilon}, \\
f^{-}=f_{0}+C_{2} \mathrm{e}^{z / l}-e E v_{x} \tau\left(1-\mathrm{e}^{z / l}\right) \frac{\partial f_{0}}{\partial \varepsilon} .
\end{gathered}
$$

Here $l=v_{z} \tau$ and $C_{1}, C_{2}$ are certain functions of velocity determined by joining conditions of the electron wave functions at $z=0$ and $z=z_{0}$.

In the case of an electron transport along the interface, the electric field is a constant in the region $0<z<z_{0}$, and the longitudinal conductivity is determined by the mean value of the current

$$
\bar{j}_{x}=\frac{1}{z_{0}} \int_{0}^{z_{0}} j_{x}(z) \mathrm{d} z
$$

where

$$
j_{x}(z)=2 e \int \frac{\mathrm{d}^{3} k}{(2 \pi)^{3}} v_{x}\left(f^{+}+f^{-}\right) .
$$

Using formulas (2), for the integrals in (3) and (4) one obtains:

$$
\begin{gathered}
\int_{0}^{z_{0}}\left(\tilde{f}^{+}+\tilde{f}^{-}\right) \mathrm{d} z= \\
=-l \tilde{C}_{1}\left(\mathrm{e}^{-z_{0} / l}-1\right)+l \tilde{C}_{2}\left(\mathrm{e}^{z_{0} / l}-1\right)+2 E v_{z} \tau\left(z_{0}-l \sinh \left(z_{0} / l\right)\right),
\end{gathered}
$$

where the new constants

$$
C_{1}=\left(-e \frac{\partial f_{0}}{\partial \varepsilon}\right) \tilde{C}_{1}, \quad C_{2}=\left(-e \frac{\partial f_{0}}{\partial \varepsilon}\right) \tilde{C}_{2}
$$

are introduced. Their values should be determined for a specific structure of interfaces between the layers.

Apart from the conditions of periodicity for the functions $f^{ \pm}$

$$
f^{ \pm}( \pm 0)=f^{ \pm}\left(z_{0} \pm 0\right)
$$

the conditions of joining at any interface are also imposed. 
When obtaining the equations of sewing, we follow the method proposed in [1] for a problem of electron scattering at a rough metal-vacuum interface and worked out in [2] for the problem of conductivity of a contact of two metals.

At the interface, the carriers are quantum reflected and scattered from defects. Accounting for elasticity of these processes, the wave function in the region $0<$ $z<z_{0}$ can be written as a superposition of states on the isoenergetic surface:

$$
\Psi_{1}(\varepsilon, \rho)=\int \frac{\mathrm{d} k_{z} d^{2} k_{\rho}}{(2 \pi)^{3}} a_{1}\left(k_{z}, \mathbf{k}_{\rho}\right) \mathrm{e}^{\mathrm{i}\left(k_{z} z+\mathbf{k}_{\rho} \cdot \rho\right)} \delta\left(\varepsilon-\frac{\left(k_{z}^{2}+k_{\rho}^{2}\right)}{2 m}\right),
$$

where $\rho=(x, y)$ is a vector perpendicular to the axis of the system. After evaluating the integral over $k_{z}$, we obtain the expression:

$$
\Psi_{1}(\varepsilon, \rho)=\int \frac{\mathrm{d}^{2} k}{(2 \pi)^{2}} \frac{1}{v^{\prime}}\left[a_{1 \mathbf{k}}^{>} \mathrm{e}^{\mathrm{i} k_{z}^{\prime} z}+a_{1 \mathbf{k}}^{<} \mathrm{e}^{-\mathrm{i} k_{z}^{\prime} z}\right] \mathrm{e}^{\mathrm{i} \mathbf{k} \cdot \rho},
$$

where the notations $\mathbf{k} \equiv \mathbf{k}_{\rho}, \quad k_{z}^{\prime}=\sqrt{2 m \varepsilon-k^{2}}, \quad v^{\prime}=k_{z}^{\prime} / m$ are introduced (from here on we put $\hbar=1)$. The upper indices $(>,<)$ refer to two possible signs of $k_{z}^{\prime}$. Therefore, $a_{1 \mathbf{k}}^{>}$and $a_{1 \mathbf{k}}^{<}$are amplitudes of the waves moving in the opposite directions.

For the wave function in the region $z_{0}<z<2 z_{0}$, we obtain an analogous expression:

$$
\Psi_{2}(\varepsilon, \rho)=\int \frac{\mathrm{d}^{2} k}{(2 \pi)^{2}} \frac{1}{v^{\prime}}\left[a_{2 \mathbf{k}}^{>} \mathrm{e}^{\mathrm{i} k_{z}^{\prime} z}+a_{2 \mathbf{k}}^{<} \mathrm{e}^{-\mathrm{i} k_{z}^{\prime} z}\right] \mathrm{e}^{\mathrm{i} \mathbf{k} \cdot \rho} .
$$

The Lagrangian of the system of two layers divided by a barrier has the form:

$$
\begin{gathered}
L=\int_{0}^{2 z_{0}} \mathrm{~d} z \mathrm{~d}^{2} \rho\left\{\frac{\left|\nabla \Psi_{1}\right|^{2}}{2 m}\left[1-\Theta\left(z-z_{0}\right)\right]-\frac{\left|\nabla \Psi_{2}\right|^{2}}{2 m} \Theta\left(z-z_{0}\right)\right. \\
\left.-\varepsilon\left|\Psi_{1}\right|^{2}\left(1-\Theta\left(z-z_{0}\right)\right)+\varepsilon\left|\Psi_{2}\right|^{2} \Theta\left(z-z_{0}\right)+V(\rho)\left|\Psi\left(z_{0}, \rho\right)\right|^{2} \delta\left(z-z_{0}\right)\right\},
\end{gathered}
$$

where $V(\rho)$ is a scattering potential at the interface, $\Theta(z)$ is a step function, $\Psi=\Psi_{1}$ at $z<z_{0} ; \Psi=\Psi_{2}$ at $z>z_{0} ; \Psi=\Psi_{1}, \Psi_{2}$ at $z=z_{0}$. After a variation over the variable $\Psi$ in (11) we obtain:

$$
\begin{gathered}
-\frac{1}{2 m} \partial_{i}\left(1-\Theta\left(z-z_{0}\right)\right) \partial_{i} \Psi_{1}-\frac{1}{2 m} \partial_{i} \Theta\left(z-z_{0}\right) \partial_{i} \Psi_{2} \\
+\delta\left(z-z_{0}\right) V(\rho) \Psi-\varepsilon \Psi=0 .
\end{gathered}
$$

The integration of (12) over an infinitely small region $\delta \rightarrow+0$ near the point $z=z_{0}$ gives an equation of joining for wave functions:

$$
\left.\frac{1}{2 m} \frac{\mathrm{d} \Psi_{1}}{\mathrm{~d} z}\right|_{z=z_{0}-\delta}-\left.\frac{1}{2 m} \frac{\mathrm{d} \Psi_{2}}{\mathrm{~d} z}\right|_{z=z_{0}+\delta}+\left.V(\rho) \Psi\right|_{z_{0}}=0
$$


which, after substitution of formulas (9) and (10), can be rewritten in the form:

$$
a_{1 \mathbf{k}}^{>}-a_{1 \mathbf{k}}^{<}+a_{2 \mathbf{k}}^{<}-a_{2 \mathbf{k}}^{>}-2 \mathrm{i} \sum_{\mathbf{q}} \frac{V_{\mathbf{q}}}{v^{\prime}}\left(a_{1 \mathbf{k}-\mathbf{q}}^{>}+a_{1 \mathbf{k}-\mathbf{q}}^{<}\right)=0 .
$$

The second equation for the amplitudes is a consequence of the continuity condition for wave functions $\Psi_{1}\left(z_{0}, \rho\right)=\Psi_{2}\left(z_{0}, \rho\right)$ at the interfaces

$$
a_{1 \mathbf{k}}^{>}+a_{1 \mathbf{k}}^{<}=a_{2 \mathbf{k}}^{>}+a_{2 \mathbf{k}}^{<} .
$$

The equations for distribution functions follow from their relation to the diagonal components of the density matrix

$$
\left|a_{1}^{<}\right|^{2}=v f^{-}, \quad\left|a_{1}^{>}\right|^{2}=v f^{+},
$$

and from analogous expressions for the second region $z_{0}<z<2 z_{0}$ (factor $v$ in these formulas is related to the normalization conditions).

Further we consider two cases corresponding to different model descriptions of the interface between the conducting layers.

For the tunnelly transparent barrier (no impurities or defects at the interface when $V(r)=$ const), from formulas (14)-(16) there follows the result:

$$
\left\{\begin{array}{l}
f^{-}\left(z_{0}-0\right)=R f^{+}\left(z_{0}-0\right)+(1-R) f^{-}\left(z_{0}+0\right) \\
f^{+}\left(z_{0}+0\right)=R f^{-}\left(z_{0}+0\right)+(1-R) f^{+}\left(z_{0}-0\right)
\end{array}\right.
$$

where $R(v)$ is a reflection coefficient at a barrier. Substituting the values of functions $f^{ \pm}$from (2) in (17) and taking into account their periodicity (7), we obtain a set of equations for the determination of the coefficients $\tilde{C}_{1}, \tilde{C}_{2}$. Solving the obtained set of equations we become assured that in the absence of scattering from the interface $\tilde{C}_{1}=\tilde{C}_{2}=E v_{x} \tau$. Using this value in (3)-(5), after integration we obtain an expression for the longitudinal conductivity:

$$
\sigma_{\|}^{(1)}=\sigma_{0}=\frac{e^{2} n \tau}{m},
$$

where $\sigma_{0}$ is a bulk conductivity of the material of the conducting layers.

In the presence of impurities or defects at the interface we assume the corresponding random potential to be Gaussian:

$$
\langle V(\rho)\rangle=0, \quad\left\langle V(\rho) V\left(\rho^{\prime}\right)\right\rangle=\gamma \delta\left(\rho-\rho^{\prime}\right),
$$

where the angled brackets refer to the averaging over realizations of the random potential. In this case the equations of sewing at the interface have the form:

$$
\begin{aligned}
f_{k}^{-}\left(z_{0}-0\right)= & f_{k}^{-}\left(z_{0}+0\right)\left(1-\frac{4 \pi \gamma}{v} \sum_{\mathbf{q}} \frac{1}{v^{\prime}}\right)+ \\
& +\frac{2 \pi \gamma}{v} \sum_{\mathbf{q}} \frac{1}{v^{\prime}} f_{k^{\prime}}^{+}\left(z_{0}-0\right)+\frac{2 \pi \gamma}{v} \sum_{\mathbf{q}} \frac{1}{v^{\prime}} f_{k^{\prime}}^{-}\left(z_{0}+0\right), \\
f_{k}^{+}\left(z_{0}+0\right)= & f_{k}^{+}\left(z_{0}-0\right)\left(1-\frac{4 \pi \gamma}{v} \sum_{\mathbf{q}} \frac{1}{v^{\prime}}\right)+ \\
& +\frac{2 \pi \gamma}{v} \sum_{\mathbf{q}} \frac{1}{v^{\prime}} f_{k^{\prime}}^{+}\left(z_{0}-0\right)+\frac{2 \pi \gamma}{v} \sum_{\mathbf{q}} \frac{1}{v^{\prime}} f_{k^{\prime}}^{-}\left(z_{0}+0\right),
\end{aligned}
$$


where index $k$ corresponds to the z-component of the momentum, whereas the sum over $q$ means summation over the longitudinal components of the momentum (i.e. along the interface).

Substituting in (20) the boundary values of the distribution functions from (2) we get for the coefficients $\tilde{C}_{1}, \tilde{C}_{2}$ :

$$
\tilde{C}_{1}=E l_{x}\left(1-\frac{2 \tilde{\gamma}\left(v_{\mathrm{F}} / v\right)}{1-\mathrm{e}^{-z_{0} / l}}\right), \quad \tilde{C}_{2}=E l_{x}\left(1+\frac{2 \tilde{\gamma}\left(v_{\mathrm{F}} / v\right)}{1-\mathrm{e}^{z_{0} / l}}\right),
$$

where the dimensionless scattering parameter $\tilde{\gamma}=2 m^{2} \gamma$ is introduced.

Using (21) in (3)-(5) for the electric current, we obtain an expression for conductivity in the presence of electron scattering from the nonidealities of the interface:

$$
\sigma_{\|}^{(2)}=\sigma_{0}\left(1-\frac{3 \tilde{\gamma} l_{\mathrm{F}}}{2 z_{0}} \Phi(\xi)\right),
$$

where

$$
\begin{gathered}
\Phi(\xi)=\int_{0}^{1}\left(1-y^{2}\right)\left(1-\mathrm{e}^{-\xi / y}\right) \mathrm{d} y=\frac{2}{3}-\xi \Gamma(-1, \xi)+\xi^{3} \Gamma(-3, \xi), \\
\xi=z_{0} / l_{\mathrm{F}},
\end{gathered}
$$

and $\Gamma(a, \xi)$ is an incomplete gamma-function [3]. Note that the function $\Phi(\xi)$ has the following asymptotic properties: $\Phi(\xi) \approx \xi \ln (1 / \xi)$ at $\xi<<1$, and $\Phi(\xi) \approx 2 / 3$ at $\xi>>1$.

Summarizing, we would like to make several remarks on the received results. The first of them refers to the applicability of the expressions. It was assumed everywhere above that the dimensionless scattering parameter $\tilde{\gamma}$ is small, so that the condition $\tilde{\gamma} l / z_{0}<<1$ holds. Then, as it is seen from (18), for smooth tunnel barriers the conductivity of the structure coincides with $\sigma_{0}$, which is a consequence of specular scattering at the boundaries of the conducting layer. At the same time, the presence of impurity scattering at the interface leads to a dependence of the structure conductivity upon the parameter $l / z_{0}$. An additional factor pointing to the nonideality of the interfaces is a change in the character of the dependence $\Phi(\xi)$ at $l / z_{0} \sim 1$ 


\section{References}

1. Falkovsky L.A. Transport phenomena at metal surfaces. // Adv. Phys., 1983, vol. 32, No. 5, p. $753-789$.

2. Dugaev V.K., Litvinov V.I., Petrov P.P. Electric-current transmission through the contact of two metals. // Phys. Rev. B., 1995, vol. 52, No. 7, p. 5306-5312.

3. Abramowitz M., Stegun I.A. Handbook of Mathematical Functions. Moscow, Nauka, 1979 (in Russian).

\section{Провідність у багатошарових структурах з сильно виродженим електронним газом}

\section{П.П.Петров}

Державний університет "Львівська політехніка", 290646 Львів, вул. С.Бандери, 12

Отримано 31 серпня 1998 р.

Обчислена електропровідність періодичних багатошарових структур з виродженим електронним газом. Показано, що для гладких тунельно прозорих бар'єрів опір структури співпадає з відповідним значенням для об'ємного матеріалу. У випадку, коли на границях розділу існує випадковий розсіюючий потенціал, провідність системи зменшується зі збільшенням відношення довжини вільного пробігу електронів до періоду структури.

Ключові слова: багатошарова структура, провідність.

PACS: $73.20 . H, 82.65 . Y$ 